\title{
Familial Hypodontia: A Case Series
}

\author{
Tangade SP, Ravishankar TL, Batra M, Shah AF
}

\begin{abstract}
${ }^{1}$ Department of Public Health Dentistry
Kothiwal Dental College \& Research Centre

Kanth Road, Moradabad-244001, Uttar Pradesh, India.

\section{ABSTRACT}

Corresponding Author

Pradeep S. Tangade

Department of Public Health Dentistry

Kothiwal Dental College \& Research Centre

Kanth Road, Moradabad-244001, Uttar Pradesh, India.

E-mail: ptangade@rediffmail.com

The phenomenon of congenitally missing teeth has been described with different names: hypodontia, oligodontia, anodontia, congenitally missing teeth and dental agenesis. The present paper reports familial hypodontia, involving two siblings from a single family. Case I reported the absence of 18, 12, 22 and 31 teeth whereas case II reported the absence of 31 and 41 . On the evaluation of the two reported cases that were offspring of same parents and absence of any clinical features associated with any syndrome the final diagnosis of non- syndromic familial hypodontia was made. Both the cases presented agenesis of the teeth leading to malocclusion and aesthetic disturbances. Cases were offered a treatment of interdisciplinary dental approach.
\end{abstract}

\section{KEY WORDS}

\section{Citation}

Tangade SP, Ravishankar TL, Batra M, Shah AF. Familial Congenital absence, familial, hypodontia, incisor, tooth agenesis. Hypodontia: A Case Series. Kathmandu Univ Med J 2015;50(2):167-9.

\section{INTRODUCTION}

Smile is one of the important facial expressions for mankind and teeth have a key role to play. Missing teeth have a wide array of consequences. The term used for describing the phenomenon of congenitally missing teeth is hypodontia, whereas a large number of missing teeth is defined as Oligodontia and the complete absence of teeth is defined as anodontia. ${ }^{1}$

The prevalence of tooth agenesis (excluding third molars) in the general population differs according to geographical area and gender, and ranges from 3.2 to $5.5 \%$ for men and from 4.6 to $7.6 \%$ for women. ${ }^{2}$ The mandibular second premolar is the tooth most commonly affected (2.9-3.2\%), followed by the maxillary lateral (1.6-1.8\%), and the maxillary second premolar (1.4-1.6\%). ${ }^{3}$

Several etiological factors have been suggested for the development failure of the permanent tooth germ, thus leading to its absence, such as: physical obstruction, dental lamina rupture, limitation of space or functional anomalies. The development of human dentition in terms of structure and organization is under genetic control and environmental factors. ${ }^{4}$ Two mutations causing isolated tooth agenesis have been identified. A point mutation in the MSX9 gene was identified in affected members of a family with missing second premolars and third molars. ${ }^{5}$ Hypodontia can be associated with several syndromes like, ectodemal dysplsia, Rieger syndrome, orodigitofacial dysostosis and Witkop tooth-nail syndrome.

\section{CASE REPORTS}

Two male patients 17 year old and 18 year-old siblings reported to the Department of Public Health Dentistry for a routine dental check-up. Both the subjects were having hypodontia and after thorough history and examination two cases of familial hypodontia were recorded.

\section{Case 1:}

An 18-year male came for first dental visit with no prior experience of having undergone any dental procedure. Medical history was non-contributory. The patient was the eldest of six siblings born to parents of a nonconsanguineous marriage. The patient was of moderate build and height. Extra oral examination revealed a face with normal facial profile and normal skeletal dental base 
relations. The consent was taken from the patient prior to the detailed examination.

Intra oral examination revealed the absence of the maxillary permanent lateral incisors bilaterally, mandibular permanent central incisor of third quadrant and third molar of first quadrant. Presence of a retained primary canine and a decayed primary lateral incisor in second quadrant was also observed [Fig. 1].

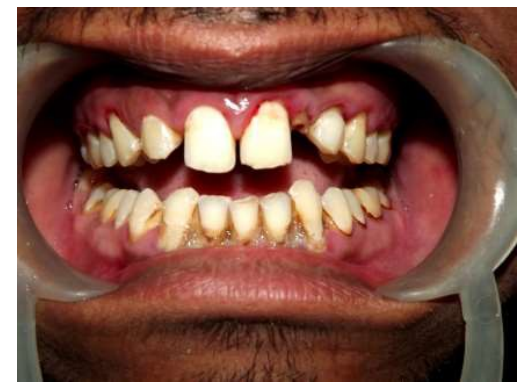

Figure 1. Showing missing permanent right lateral incisor, left lateral incisor and left third molar in maxillary arch and missing mandibular right central incisors.

An Orthopantomogram (OPG) confirmed the absence of 18, 12, 22 and 31. The increased spacing in the roots of the central incisors and canine in first quadrant indicated a missing lateral incisor. In second quadrant there was presence of a decayed primary lateral incisor with partially resorbed root and pathologic periapical changes. A retained primary canine with smaller and distally displaced roots distal to the decayed primary incisor highlighted the absence of the lateral incisors and an impacted permanent canine was present $3 \mathrm{~mm}$ above the root apex of premolars with crown overlapping the root apex of 21 and root apex projecting towards the base of the maxillary sinus. Radiograph also displayed congenital absence of third molar in the first arch. Other findings in the orthopantomogram were within normal limits considering the patient's age [Fig. 2].

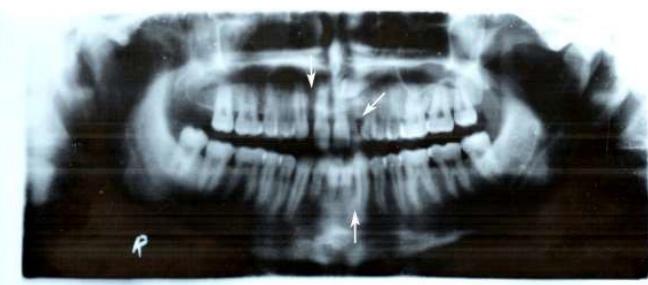

Figure 2. Orthopantomogram of case 1 , showing absence of 12 , 22,31 \& 18 and increased spacing in central incisors and canine in first quadrant, presence of a decayed primary lateral incisor, retained primary canine distal, impacted permanent canine.

The treatment plan considered for the patients included extraction of the decayed primary lateral incisor followed by root canal treatment of retained primary canine in the second arch. Due to the poor socioeconomic status patient rejected the decision of orthodontic treatment for impacted canine, thus open extraction (deimpactiion) of the impacted canine was suggested without any iatrogenic trauma to 21 . Post healing fixed prosthodontic treatment was advised.

\section{Case 2:}

A 17-year male, younger brother of the patient presented as case 1 also exhibited missing permanent mandibular central incisors and maxillary third molars bilaterally [Fig. 3].

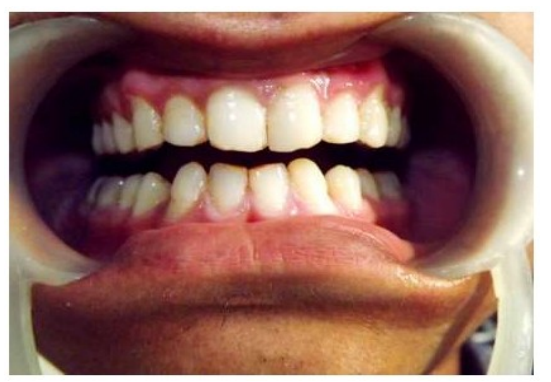

Figure 3. Showing missing permanent right and left mandibular central incisors.

Extra oral examination revealed a face with normal facial profile and normal skeletal dental base relations. There was absence of any clinical feature like spacing in the mandibular anterior teeth or rotations of any teeth present. Radiographic examination confirmed the congenital absence of all missing teeth [Fig. 4].

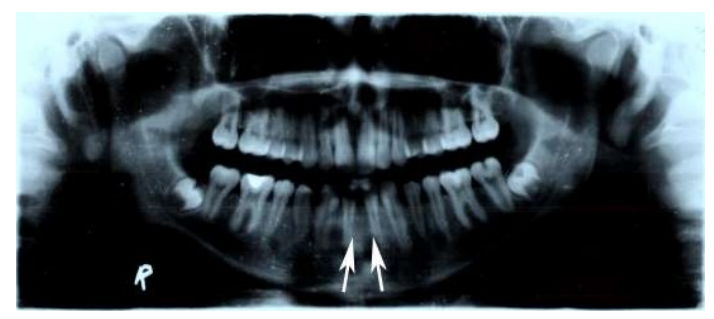

Figure 4. Orthopantomogram of case 2 showing absence of 31 and 41 and absence of maxillary third molars.

On general examination there were no positive findings related to any syndromes associated with hypodontia. No treatment was advised for the case 2 as there was absence of spacing or rotations in the anterior teeth. All teeth were in proper alignment and presented normal coronal morphology. Complete set of investigations were done and the final diagnosis on the basis of examination and results of investigations was formulated as non-syndromic form of hypodontia.

On the evaluation of the two reported cases that were offspring of same parents and absence of any clinical features associated with any syndrome the final diagnosis of non- syndromic familial hypodontia was made. 


\section{DISCUSSION}

Hypodontia is most commonly used in describing the phenomenon of congenitally missing teeth. ${ }^{6}$ It can be classified as isolated or non-syndromic and syndromic hypodontia. Hypodontia is associated with several syndromes however this anomaly can also be present without any associated syndrome or systemic disease. ${ }^{7}$ The present case is a classic example of non syndromic familial hypodontia.

The biologic basis for the congenital absence of permanent teeth is partially explained by the failure of the lingual or distal proliferation of the tooth bud cells from the dental lamina. The causes of hypodontia are attributed to environmental factors such as irradiation, tumours, trauma, hormonal influences, rubella, and thalidomide or to hereditary genetic dominant factors, or to both. ${ }^{8}$

Mutations in transcription factors MSX1 and PAX9 have been identified in families with an autosomal dominant oligodontia. A missense mutation was first found by the Vastardis group in the meodomain of MSX1 gene in chromosome 4 (4p16) in all affected members of a family with missing second premolars and third molars as a prominent feature. ${ }^{9}$

Although tooth agenesis is occasionally caused by environmental factors, in the majority of cases hypodontia has a genetic basis. In familial hypodontia, the type of inheritance in the majority of families seems to be autosomal dominant with incomplete penetrance and variable expressivity. ${ }^{10}$ An autosomal recessive model of inheritance is also possible. Sex-linked inheritance patterns and a polygenic or multifactorial model of inheritance have also been suggested. ${ }^{10}$

The reduced number of lateral incisors is of particular significance for aesthetics. In most cases, the necessity of performing orthodontic-prosthetic treatment is a basic requirement, especially for the patients combining hypodontia with changes in the shape and size of available teeth and dental-jaw discrepancy resulting in diastema. ${ }^{11}$

The role of genetic factors in the aetiology of various dental anomalies including alteration in number of teeth is been studied in depth by the researchers and such kind of reports help in giving the support to the genetic theory of disease expression. ${ }^{12}$ Aubrey Chosack suggests that it is possible that different types of hypodontia are caused by different genetic factors. ${ }^{13}$ If hypodontia resulted from an autosomal recessive gene one would expect about $25 \%$ of siblings to be affected. If hypodontia was an autosomal dominant trait with full penetrance, one would expect about $50 \%$ of siblings to be affected.

8. Mostowska A, Biedziak B, Trzeciak WH. A novel mutation in PAX9 causes familial form of molar oligodontia. Eur J Hum Genet 2006; 14: 173-9.

9. Lammi L, Halonen K, Pirinen S, Thesleff I, Arte S, Nieminen P. A missense mutation in PAX9 in a family with distinct phenotype of oligodontia. Eur J Hum Genet. 2003;11(11):866-71.

10. Arte S, Pirinen S. Hypodontia. Orphanet encychlopedia, www.orpha. net/data/GB/UK- hypodontia.pdf. 2007; 1-7.

11. Krumova V, Yoncheva I. Hypodontia of the upper lateral incisors - a therapeutic approach and factors, which have determined it (a clinical case). Journal of IMAB - Annual Proceeding (Scientific Papers) 2008; 14(2): 55-8.

12. Agarwal A, Gundappa M, Miglani S, Nagar R. Asyndromic hypodontia associated with tooth morphology alteration: A rare case report. Journal of Conservative Dentistry: JCD. 2013;16(3):269-71.

13. Chosack, Eidelman, \& Cohen, Hypodontia: A Polygenic Trait, J Dent Res 1975; 54(I):16-9.

7. Shinh AS, Goyal D, Shaik JA, Guram G. Familial Tendency Of Oligodontia In Three Generations: A Rare Case Report. IJDS 2014; 2(6):72-6. 